\title{
Diseño e implementación de un modelo de madurez de capacidades logísticas en servicios de atención médica domiciliaria
}

\author{
Design and Implementation of a Logistic Capability Maturity Model for Health Care Services at Home \\ Desenho e implementação de um modelo de maturidade de capacidades logísticas em serviços de atenção \\ médica domiciliar
}

Elena Valentina Gutiérrez ${ }^{\mathrm{a}}$

Universidad de Antioquia, Colombia

elena.gutierrez@udea.edu.co

ORCID: http://orcid.org/0000-0003-1139-735X

Sebastiá Cortés Zapata

Universidad de Antioquia, Colombia

ORCID: http://orcid.org/0000-0001-7614-8819

Juan Sebastiá Jaén Posada

Universidad de Antioquia, Colombia

ORCID: http://orcid.org/0000-0001-7077-5109
DOI: https://doi.org/10.11144/Javeriana.rgps17-35.dimm Redalyc: http://www.redalyc.org/articulo.oa?id=54557477009

Fecha de recepción: 12 Julio 2017

Fecha de aprobación: 11 Mayo 2018 Fecha de publicación: 01 Noviembre 2018

\section{Resumen:}

La prestación de servicios de atención médica domiciliaria (HHC) implica decisiones logísticas en el diseño, la implementación y el control del servicio. Muchas instituciones que prestan servicios de HHC en Colombia toman dichas decisiones de manera empírica y pocas veces se utilizan métodos cuantitativos para apoyarlas de manera sistematizada. Esta situación genera la necesidad de caracterizar el nivel de madurez de las capacidades logísticas de las instituciones que prestan servicios de HHC, con el objetivo de identificar brechas tecnológicas y posibles caminos de mejoramiento. En este trabajo se presenta el diseño de un modelo de madurez de capacidades logísticas en servicios de HHC, basado en el Capability Maturity Model (CMM). El modelo es implementado en una institución de salud habilitada para la prestación de servicios de HHC en Antioquia, Colombia. Los resultados evidencian las brechas existentes y la utilidad del modelo para identificar oportunidades de mejora a través de métodos cuantitativos.

Palabras clave: servicios de atención de salud a domicilio, gestión en salud, modelo de madurez de capacidades.

\section{Abstract:}

Health Care Services at Home (HCSH) implies to make logistic decisions about the design, implementation and control of the services. Many institutions providing HCSH services in Colombia make these decisions empirically and in quite few times they are backed by quantitative methods in a systematic way. This situation creates the need to characterize the maturity levels of the logistic capability in the institutions providing HCSH aiming to identify the technological gaps and potential paths for improvement. This work provides the design of a logistic capability maturity model for the HCSH services based on the Capability Maturity Model (CMM). The model is implemented in a health institution set up to provide HCSH services in the Antioquia Province, Colombia. The results show the existing gaps as well as the model usefulness to identify the opportunities for improvement thanks to quantitative methods.

Keywords: health care services at home, health management, Capability Maturity Model.

\section{Resumo:}

A prestação de serviços de atenção médica domiciliar (HHC) implica decisões logísticas no desenho, implementação e controle do serviço. Muita instituição que presta serviços de HHC na Colômbia toma tais decisões de forma empírica e raras vezes são utilizados métodos quantitativos para apoiá-las de maneira sistematizada. Tal situação gera necessidade de caracterizar o nível de maturidade das capacidades logísticas das instituições que prestam serviços de HHC, com o objetivo de identificar lacunas tecnológicas e possíveis caminhos de melhoramento. Neste trabalho apresenta-se o desenho de um modelo de maturidade de capacidades logísticas em serviços de HHC, baseado no Capability Maturity Model (CMM). O modelo é implementado em uma instituição de saúde 
habilitada para a prestação de serviços de HHC em Antioquia, Colômbia. Os resultados evidenciam as lacunas existentes e a utilidade do modelo para identificar oportunidades de melhora através de métodos quantitativos.

Palavras-chave: serviços de atenção de saúde a domicílio, gestão em saúde, modelo de maturidade de capacidades.

\section{Introducción}

Los servicios de atención médica domiciliaria (HHC por sus siglas en inglés, home health care), se han convertido en un componente importante de los sistemas de salud en el mundo $(1,2)$. En Colombia, han servido inicialmente como una alternativa de atención geriátrica durante la década de los noventa (2, $3)$, para luego convertirse en un modelo de negocio $(4,5)$ alternativo a la escasez de camas hospitalarias, capaz de reducir costos y mejorar la calidad de vida del paciente y su núcleo cercano $(6,7)$. En las tres ciudades más grandes de Colombia, el número de instituciones habilitadas que ofrecen este servicio ha venido creciendo de manera rápida $(3,6)$. Solamente en el valle de Aburrá, región del centro-sur del departamento de Antioquia, Colombia, existían en febrero del 2017, 34 instituciones que proveían este servicio de manera tercerizada a hospitales y aseguradoras (8). A la misma fecha, el número total de instituciones habilitadas para presentar estos servicios en Colombia era de 666, las cuales atendían pacientes crónicos sin ventilación mecánica (8). A la par del crecimiento de las instituciones habilitadas, también ha venido aumentando el número de publicaciones nacionales e internacionales que estudian y proponen mejoras a diferentes aspectos de esta alternativa de atención (9). Los recientes avances en telemedicina, con el diseño de dispositivos de cuidado y tratamiento remoto $(10,11,12)$, vaticinan que esta modalidad de atención tendrá un mayor protagonismo dentro de los sistemas de salud del país (1).

El HHC se define como el conjunto de servicios médicos de atención de carácter ambulatorio realizados en el hogar bajo la supervisión profesional, con el fin de brindar continuidad a un tratamiento previo intrahospitalario $(1,3,6)$. Por definición, este servicio se presta por fuera del entorno hospitalario, luego su proceso asistencial está estrechamente vinculado al desempeño de procesos logísticos, ligados principalmente al transporte de personal asistencial, a los insumos médicos y equipos, así como al almacenamiento y el control de estos. Los procesos logísticos deben garantizar que como mínimo el paciente sea atendido en el momento que lo necesita, recibiendo los insumos que su tratamiento requiere (9). Para que esto sea posible con un nivel de desempeño de calidad y competitivo, es necesario que el diseño, la ejecución, la revisión y el mejoramiento de estos procesos logísticos sean rigurosos y continuos, y que se cuente con las herramientas para llevarlos a cabo. En Colombia, el Ministerio de Salud y Protección Social reglamenta todos los requisitos mínimos que cada institución prestadora de servicios de salud (IPS) necesita para ser habilitada (13). Esta resolución incluye también a las instituciones que prestan el servicio de HHC, en la cual se definen estándares claros de capacidad técnico-administrativa, suficiencia patrimonial y financiera y capacidad tecnológica científica. La resolución es una herramienta que establece el estándar y permite identificar las falencias y ventajas de cada IPS en sus procesos asistenciales. No obstante, el HHC es un tipo de servicio diferente, en el que el paciente no se desplaza a la institución (14), y depende intrínsecamente de un componente logístico el cual no es explícitamente regulado en Colombia como el componente asistencial. Aquí es donde se identifica una brecha. Si bien existe literatura que define las necesidades y los problemas logísticos de este tipo de atención $(9,15,16)$, aún hacen falta trabajos que permitan definir y evaluar el grado de madurez en que se encuentran estos procesos en la atención domiciliaria de los pacientes.

Como se propone en el marco conceptual presentado en (16), las capacidades logísticas para la prestación de servicios de HHC pueden definirse como el conjunto de decisiones que se deben tomar en cada una de las funciones logísticas. Dicho marco conceptual define los procesos logísticos de acuerdo con las funciones logísticas, y se basa en trabajos de la literatura que revisan decisiones de ese tipo (17, 18, 19, 20, 21, 22, 23). 
De igual forma, el marco conceptual deAne los procesos de servicio como el conjunto de pasos que se llevan a cabo desde que un paciente es remitido al sistema de HHC hasta que sale de él. Una revisión de la literatura evidencia que solo el trabajo presentado en (6) hace una medición de las capacidades logísticas de las IPS habilitadas para prestar servicios de HHC en una región de Colombia. Una de las formas en las que se ha evaluado el grado de madurez de los procesos es el Modelo de Madurez de Capacidades (CMM por sus siglas en inglés, Capability Maturity Model). El CMM es una estructura conceptual que tiene como fin mejorar la gestión y el desarrollo de procesos de una organización, de manera consistente y disciplinada (24). El modelo tuvo sus inicios en 1986 cuando el Departamento de Defensa de los Estados Unidos solicitó al Instituto de Ingeniería de Software que desarrollara un marco para evaluar a los proveedores de software y ayudar a las organizaciones a mejorar sus procesos. Fue en 1993 cuando el marco evolucionó al Modelo de Madurez de Capacidades, que establece un conjunto procesos clave que se agrupan en áreas clave de proceso, y que según el estado, la organización y el grado de desarrollo que hayan alcanzado, se pueden agrupar en uno de los cinco niveles de madurez que representa el modelo (25). La literatura evidencia que el CMM se ha utilizado ampliamente en diversas aplicaciones de producción de bienes y servicios, las cuales incluyen la evaluación de procesos en el diseño de software, el diseño en ingeniería, la gestión de negocios, las ciencias sociales, las ciencias de la decisión, la agricultura, el medio ambiente y la salud (26).

De este modo, el propósito de este trabajo es presentar un instrumento que permita a las IPS habilitadas para la prestación de servicios de HHC definir y evaluar sus capacidades logísticas, con el propósito de mejorar la prestación del servicio. Así, la pregunta de investigación de este trabajo es: ¿cómo medir el nivel de madurez de las capacidades logísticas de las instituciones que prestan servicios de HHC, con el objetivo de identificar brechas tecnológicas y posibles caminos de mejoramiento? El documento se encuentra organizado de la siguiente forma: primero, en la siguiente sección se presentan los métodos y materiales, donde se describen las cuatro etapas en las que se desarrolló la investigación. Luego, se presentan los resultados y se hace un análisis de los hallazgos obtenidos con la implementación del modelo en un caso de estudio real. En la sección posterior se presenta la discusión, las conclusiones y se identifican las oportunidades de investigación. Finalmente, en la última sección se presentan las referencias que soportan este trabajo.

\section{Materiales y métodos}

El diseño y la implementación del modelo de madurez de capacidades logísticas para servicios de HHC se define en cuatro etapas. En la primera de estas se identifican las capacidades logísticas de una institución habilitada para la prestación de dichos servicios, a través de los procesos logísticos y los procesos de servicio. Para cada proceso, se definen las variables que inciden en la madurez de las capacidades logísticas. En la segunda etapa se diseña el instrumento estructurado que permite estimar el grado de madurez para cada uno de los procesos, basado en la adaptación de la escala del CMM, la cual se aplica a cada una de las variables previamente identificadas. En la tercera etapa se comprueba la validez y la fiabilidad del modelo de madurez. En la cuarta etapa se describe el proceso de implementación del modelo. A continuación, se hace una descripción de cada una de las cuatro etapas.

\section{Capacidades logísticas}

Con base en lo definido en (16), las capacidades logísticas para la prestación de servicios de HHC pueden definirse como el conjunto de decisiones que se deben tomar en cada una de las funciones logísticas, y como el conjunto de procesos de servicio que se llevan a cabo desde que un paciente es remitido al sistema de 
HHC hasta que sale de él. Para cada uno de estos procesos se identifica un conjunto de variables que define la madurez de las capacidades logísticas. A continuación, se presentan las capacidades logísticas en términos de dichos procesos y variables.

\section{Procesos logisticos}

Con base en el marco conceptual presentado en (16), se incluyen cuatro procesos logísticos en el modelo: (i) diseño de la red, (ii) gestión del transporte, (iii) gestión del personal asistencial y (iv) gestión de inventarios de medicamentos, equipos e insumos. En servicios de HHC, cada uno de los procesos logísticos implica un conjunto de decisiones en tres diferentes horizontes de planeación (estratégico, táctico y operativo). El diseño de la red hace referencia a las decisiones estratégicas que definen en el largo plazo la cobertura de un área geográfica mediante la localización de las instalaciones de las centrales de operaciones de HHC y la zonificación de dicha área. Por su parte, la gestión del transporte implica decisiones estratégicas, tácticas y operativas, relativas a la selección y definición del tamaño y tipo de la flota vehicular que se va a utilizar para visitar a los pacientes, a la asignación de la flota y a la secuenciación de visitas a los pacientes, mediante el diseño de rutas del personal asistencial. De igual forma, la gestión del personal asistencial implica la toma de decisiones en tres horizontes de planeación, y con ellas se define la determinación de la capacidad del sistema HHC en términos del personal asistencial, la asignación de turnos al personal y la asignación del personal a las visitas. La gestión de inventarios hace referencia a las decisiones que en los tres niveles definen respectivamente la selección de proveedores de medicamentos, suministros y equipos médicos, la definición de políticas de inventarios y el control de los inventarios, incluyendo los aspectos que inducen variabilidad al sistema. En la tabla 1 se presentan las variables evaluadas en cada uno de los procesos logísticos. 
TABLA 1

Variables evaluadas en cada proceso logístico

\begin{tabular}{|c|c|}
\hline Proceso logístico & Variables evaluadas \\
\hline Diseño de la red & $\begin{array}{l}\text { - Criterios de localización de bases de operaciones } \\
\text { - Método de zonificación del área de atención } \\
\text { - Criterios de desempeño de la zonificación } \\
\text { - Registros de demanda por zonas } \\
\text { - Equipo de trabajo para el diseño de la red } \\
\text { - Estimación del costo del diseño de la red } \\
\text { - Mejoras del diseño de la red }\end{array}$ \\
\hline $\begin{array}{l}\text { Gestión del } \\
\text { transporte }\end{array}$ & $\begin{array}{l}\text { - Naturaleza de la flota vehicular para visitas médicas } \\
\text { - Modos de transporte para visitas médicas } \\
\text { - Métodos para la selección de modos de transporte } \\
\text { - Criterios para la selección de modos de transporte } \\
\text { - Equipo de trabajo para la gestión del transporte } \\
\text { - Estimación del costo de la gestión del transporte } \\
\text { - Mejoras de la asignación dela gestión del transporte }\end{array}$ \\
\hline $\begin{array}{l}\text { Gestión del personal } \\
\text { asistencial }\end{array}$ & $\begin{array}{l}\text { - Modalidades de contratación del personal asistencial } \\
\text { - Tipos médicos de personal asistencial } \\
\text { - Métodos para deteminación de capacidad del personal asistencial } \\
\text { - Métodos para la programación de tumos de trabajo } \\
\text { - Equipo de trabajo para la gestión del personal asistencial } \\
\text { - Estimación del costo de gestión del personal asistencial }\end{array}$ \\
\hline & - Mejoras en la gestión del personal asistencial \\
\hline $\begin{array}{l}\text { Gestión de } \\
\text { inventarios }\end{array}$ & $\begin{array}{l}\text { - Tipos de inventario que gestiona la institución } \\
\text { - Métodos de selección de proveedores } \\
\text { - Métodos para definir politicas de inventario } \\
\text { - Registros de variabilidad de los niveles de inventario } \\
\text { - Equipo de trabajo para la gestión de los inventarios } \\
\text { - Estimación del costo de gestión delos inventarios } \\
\text { - Mejoras en la gestión de los inventarios }\end{array}$ \\
\hline
\end{tabular}

Fuente: elaboración propia. 


\section{Procesos de servicio}

Con base en lo definido en (16), los procesos de servicio incluidos en el modelo son: (i) remisión, (ii) admisión, (iii) programación de visitas, (iv) prestación del servicio, (v) facturación y (vi) liquidación y cartera. En servicios de HHC, cada uno de los procesos de servicio implica un conjunto de acciones que incide en la madurez de las capacidades logísticas. El proceso de remisión hace referencia a las actividades de prescripción médica y coordinación por parte del paciente, su familia, el médico y la IPS para la aceptación del servicio. En el proceso de admisión se ingresa al paciente al sistema de $\mathrm{HHC}$, a través de una validación de la prescripción médica, de los derechos administrativos del paciente de acuerdo con su entidad promotora de salud (EPS) o entidad responsable de pago, y a los aspectos logísticos y médicos como la localización geográfica del paciente y la disponibilidad de las especialidades médicas requeridas para el servicio de HHC. En el proceso de programación de visitas se ejecutan todas las acciones relativas a la asignación y secuenciación de visitas al domicilio del paciente, de modo que se cumpla con las frecuencias y los horarios definidos en la prescripción médica. El proceso de prestación de servicio hace referencia a la atención médica en sí, e implica el contacto directo del personal asistencial con el paciente. El proceso de facturación, liquidación y cartera implica la revisión y el seguimiento de las visitas realizadas, así como la generación de facturas para iniciar los procesos de cobro a las entidades responsables de pago.

Para cada uno de los procesos del servicio se identifica el conjunto de variables que inciden en la madurez de las capacidades logísticas en HHC. Las variables se ilustran en la tabla 2. 
TABLA 2

Variables evaluadas en cada proceso de servicio

\begin{tabular}{|c|c|}
\hline Proceso de servicio & Variables evaluadas \\
\hline Remisión & $\begin{array}{l}\text { - Tamaño del portafolio de servicio de atención médica domiciliaria } \\
\text { - Tipos de servicio de atención médica domiciliaria } \\
\text { - Entrega de informe de evolución médica al remitente }\end{array}$ \\
\hline Admisión & $\begin{array}{l}\text { - Medición de la capacidad de prestación de servicios } \\
\text { - Registros de demanda de pacientes atendidos y no atendidos } \\
\text { - Método para estimación de la demanda de servicios }\end{array}$ \\
\hline $\begin{array}{l}\text { Programación de } \\
\text { visitas }\end{array}$ & $\begin{array}{l}\text { - Método de programación de visitas de atención domiciliaria } \\
\text { - Criterios para la programación de visitas de atención domiciliaria } \\
\text { - Promesas de servicio de visitas de atención domiciliaria } \\
\text { - Proceso de asignación y secuenciación de visitas } \\
\text { - Criterios de asignación y secuenciación de visitas }\end{array}$ \\
\hline $\begin{array}{l}\text { Prestación del } \\
\text { servicio }\end{array}$ & $\begin{array}{l}\text { - Métodos para la prestación del servicio médico } \\
\text { - Evaluación del cumplimiento de promesas de servicio } \\
\text { - Sistema de medición de satisfacción de pacientes } \\
\text { - Estimación de tiempos de procedimientos médicos en visitas } \\
\text { - Estimación de tiempos de desplazamiento entre visitas } \\
\text { - Registro y razones de incumplimientos totales o parciales de visitas }\end{array}$ \\
\hline Facturación & - Método del proceso de facturación \\
\hline $\begin{array}{l}\text { Liquidación y } \\
\text { cartera }\end{array}$ & - Método del proceso de liquidación y cartera \\
\hline
\end{tabular}

Fuente: elaboración propia. 


\section{Diseño del modelo}

El diseño del modelo de madurez implica la construcción de un instrumento estructurado que permita estimar el grado de madurez de cada uno de los procesos de las capacidades logísticas, y por ende de la institución que preste el servicio de HHC. De este modo, y con base en los elementos definidos previamente en la literatura y en la identificación de las capacidades logísticas, se construyó un cuestionario estructurado de diez secciones que utiliza una adaptación de la escala del CMM para evaluar el grado de madurez de cada una de las variables definidas en las tablas 1 y 2.

Previamente al desarrollo de las diez secciones de la encuesta, en el cuestionario se hace una caracterización inicial de la institución a la que se evalúa su madurez de capacidades logísticas de los servicios de HHC. La caracterización incluye una descripción general de la institución, su antigüedad, modelo de negocio, tipo de grupo empresarial al que pertenece, municipios de cobertura del servicio, sistema de clasificación de pacientes y métodos de planeación estratégica. Si bien estos aspectos no se incluyen en la estimación de la métrica de la madurez, permiten situar a la institución bajo análisis en el sector.

En las secciones 1 a 4 del cuestionario, se incluye primero, para cada uno de los cuatro procesos logísticos, preguntas relativas al diseño y la operación de cada de función logística, y luego se incluye preguntas estandarizadas que indagan por los criterios de decisión, los métodos usados para la toma de decisiones, la documentación y el seguimiento de los registros, el equipo de trabajo que apoya el desarrollo y la ejecución del proceso, la estimación de los costos de la función logística y la aplicación de mejoras (variables definidas en la tabla 1).

En las secciones 5 a 10, en los seis procesos de servicio se incluye, de manera general, criterios relacionados con el número y los tipos de servicio que la IPS presta, la forma en que se mide la capacidad de los servicios, la documentación de registros de los procesos, el uso de métodos para ejecutar los procesos, la definición de promesas de servicio a los pacientes, el seguimiento de los procesos, la evolución del estado de los pacientes, actividades de realimentación y de satisfacción, el uso de los recursos para llevar a cabo los procesos y la evaluación del desempeño de los procesos (variables definidas en la tabla 2).

En la definición de la escala de madurez del modelo, a diferencia del modelo general del CMM, se definió una escala de seis niveles de madurez comparados con los cinco niveles que se han aplicado desde la ingeniería de software. El nivel adicional corresponde a un proceso con un grado de madurez cero, definido como un nivel inexistente debido a que en algunas instituciones no se cuenta o no se ha definido alguna de las variables o procesos evaluados a través del modelo de madurez propuesto en este trabajo. En la tabla 3 se presenta la escala de madurez utilizada. 
TABLA 3

Escala de madurez utilizada en el modelo

\begin{tabular}{|c|c|c|}
\hline \multicolumn{2}{|c|}{ Nivel de madurez } & \multirow{2}{*}{$\begin{array}{l}\text { Descripción } \\
\text { Proceso inexistente en la institución evaluada }\end{array}$} \\
\hline 0 & Inexistente & \\
\hline 1 & Inicial & $\begin{array}{l}\text { Se reconoce la existencia del proceso y su ejecución es ad hoc. El proceso } \\
\text { se considera apropiado, pero ocasionalmente es caótico debido a que las } \\
\text { instituciones en este nivel no disponen de un ambiente estable para el } \\
\text { desarrollo y el mantenimiento del proceso. Se evidencia falta de } \\
\text { planeación, se pueden producir fracasos, retrasos y sobrecostos. }\end{array}$ \\
\hline 2 & Repetible & $\begin{array}{l}\text { Proceso conocido, aceptado y repetido, pero no estandarizado ni } \\
\text { documentado. Se dispone de prácticas básicas de gestión de proyectos, } \\
\text { existen métricas básicas como el seguimiento delos costos y los plazos, } \\
\text { con el fin de repetir éxitos que se hayan logrado previamente. La } \\
\text { responsabilidad de la ejecución del proceso recae sobre una persona. }\end{array}$ \\
\hline 4 & Gestionado & $\begin{array}{l}\text { Se caracteriza por contar con un conjunto de métricas e indicadores de } \\
\text { calidad y productividad que se usan de modo sistemático para la toma de } \\
\text { decisiones. Además, se recogen medidas detalladas del proceso para } \\
\text { generar productos de alta calidad. El uso de herramientas de tecnología de } \\
\text { información es limitado. }\end{array}$ \\
\hline 5 & Optimizado & $\begin{array}{l}\text { El proceso es mejorado continuamente y de forma sistemática. Se hace } \\
\text { uso intensivo delas métricas, unidas a una retroalimentación cuantitativa } \\
\text { del proceso, y se gestiona el proceso de innovación. Se utilizan } \\
\text { herramientas de tecnología de información para mantener ventajas } \\
\text { competitivas. }\end{array}$ \\
\hline
\end{tabular}

Fuente: adaptado con base en (27).

La asignación del valor de madurez a cada una de las variables, de acuerdo con la escala definida, se hace de tres formas. En la primera, para las preguntas en las que se indaga por el nivel de madurez directamente, y se brindan las seis alternativas descritas en la tabla 3, el valor de madurez corresponde al valor seleccionado en la descripción de madurez del proceso. En la segunda forma se asigna la escala de madurez, linealmente, a las alternativas de la pregunta. Por ejemplo, las preguntas que tienen seis alternativas en orden ascendente, le asignan a cada alternativa, respectivamente, cada nivel de madurez. En la tercera forma se hace una asignación uniforme del nivel de madurez, de acuerdo con el número de alternativas disponible. Por ejemplo, si para el tipo de servicios asistenciales de HHC hay siete alternativas, y una institución ofrece cinco de ellas, el valor proporcional de su madurez en dicha variable será de 5/7 $(71,43 \%)$ relativo a la escala de madurez. Para calcular el valor de madurez de cada proceso logístico y de servicio, se calcula un promedio simple de cada una de las variables ilustradas en las tablas 1 y 2. 
Se construyó una interfaz gráfica en Microsoft Excel que permite sistematizar el registro de los valores obtenidos en cada una de las variables de los procesos logísticos y los procesos de servicio, y permite estimar el grado de madurez de las capacidades logísticas para los servicios de $\mathrm{HHC}$ (véase figura 1). Al igual que el cuestionario estructurado, la interfaz gráfica incluye un libro de Excel, con diez hojas de cálculo, en las que se hace el registro de los valores de madurez de cada una de las variables de los diez procesos evaluados. La interfaz tiene además dos hojas de cálculo iniciales en las que se accede al cuestionario y se hace la caracterización de la institución que se va a evaluar, y dos hojas de cálculo finales en las que se hace, respectivamente, el cálculo de los resultados de cada uno de los procesos, y se construye un mapa de madurez.

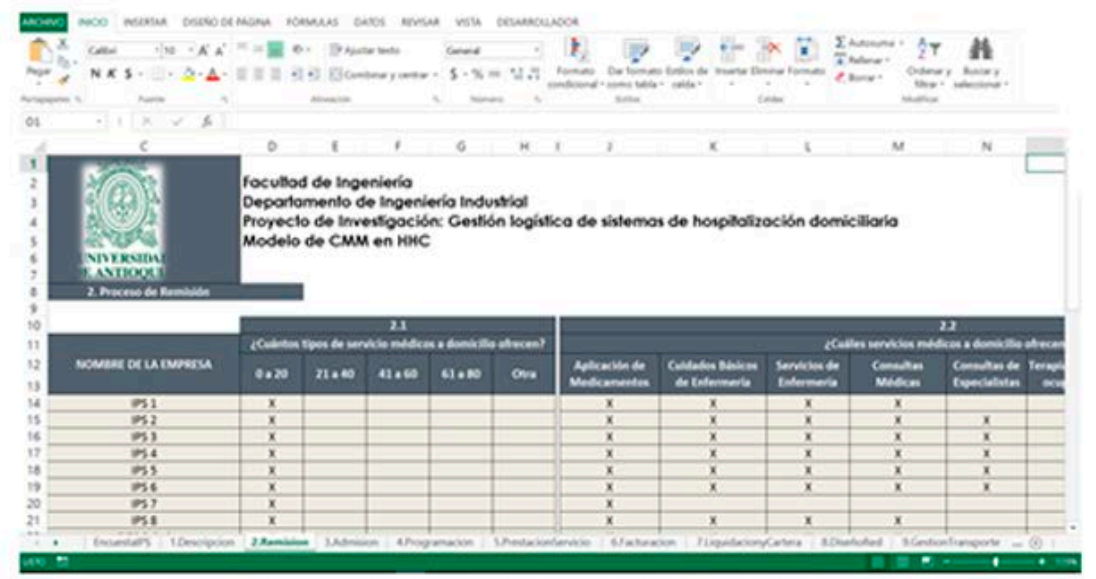

(a) Ingreso de los valores en cada una de las variables evaluadas en cada proceso

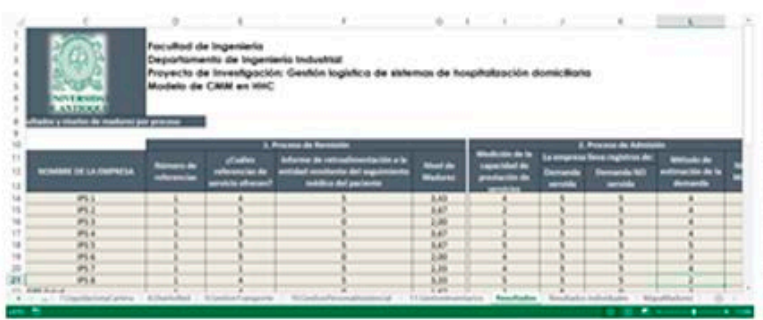

(b) Resultados de madurez

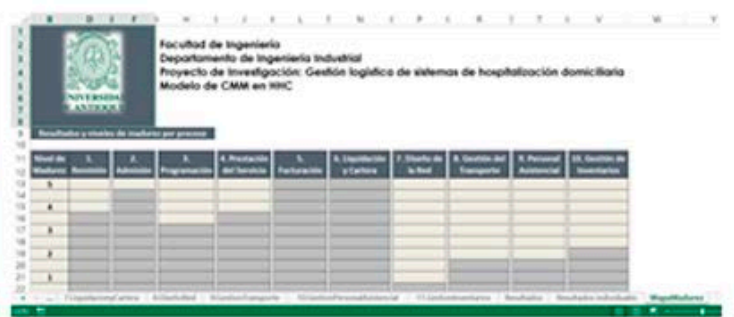

(c) Mapa de madurez

FIGURA 1

Interfaz gráfica del modelo de madurez de capacidades logísticas de servicios de HHC Fuente: elaboración propia.

\section{Validez y fiabilidad del modelo}

La validez y la fiabilidad del modelo fueron comprobadas de manera cualitativa. La primera se comprueba a través de su validez de criterio y su validez de constructo, y se basa en la validez teórica del CMM desde su creación en 1989 (27,28), así como en la validez teórica de las capacidades logísticas en HHC $(9,17,18,29,30,31,32)$. Y la fiabilidad del modelo se comprobó de dos formas. Primero, en su implementación, se hicieron múltiples entrevistas con cada uno de los cargos y roles del sistema de HHC, los cuales incluyeron el personal gerencial que toma las decisiones estratégicas, el personal administrativo que toma las decisiones tácticas y el personal operativo que ejecuta la prestación del servicio. Para cada uno de los cargos se encuestó al personal de tipo asistencial y de tipo administrativo. Segundo, se hizo una triangulación en el análisis de las variables de cada uno de los procesos, dividiendo para algunos de ellos el cuestionario en dos mitades. De este modo, se comprueba la consistencia interna del cuestionario y de los resultados de las variables y los procesos evaluados. 


\section{Implementación del modelo}

La implementación del modelo de madurez de capacidades logísticas se hizo con la IPS de la Universidad de Antioquia (IPS-UdeA). Dicha IPS es una institución prestadora de servicios de salud, que según el ránking de la revista América Economía, ha estado por varios años entre los primeros 40 hospitales de América Latina, al ofrecer un esquema de atención clínica integral a los pacientes. En la actualidad, la institución cuenta con siete sedes en el territorio colombiano, cuatro en la ciudad de Medellín, una en Barranquilla, una en San Andrés Islas y una sede en Apartadó, en el Urabá antioqueño. Entre los servicios que ofrece se encuentran ayudas diagnósticas, imagenografía, ultrasonido, fonoaudiología, diferentes especialidades de terapias, laboratorio clínico, transfusión sanguínea, hemodiálisis y el Programa de Atención Domiciliaria, entre muchos otros.

El Programa de Atención Domiciliaria (PAD) de la IPS-UdeA fue creado en el 2011, con el fin de disminuir los días de estancia hospitalaria y para mejorar el giro de cama, promoviendo la mejora de la satisfacción y la evolución del estado de los pacientes. El programa inició con los pacientes de las entidades responsables de pago que contratan con la IPS-UdeA. A la fecha, el programa cuenta con una cobertura de atención en los diez municipios del valle de Aburrá en el departamento de Antioquia, Colombia.

Asimismo, se atienden pacientes clasificados como crónicos, en su gran mayoría, pero en el momento del desarrollo del caso de estudio del PAD se trabajó con los registros de los pacientes clasificados como agudos, de los cuales se atendía en promedio a 100 pacientes por mes en el año 2015, en su mayoría del régimen subsidiado. La implementación del modelo se hizo durante el segundo semestre del 2016, en la línea del PAD que atiende pacientes agudos, la cual corresponde a una población anual promedio de 1350 pacientes. Para la implementación se recolectó información primaria en cada una de las variables de los procesos evaluados, y se realizaron múltiples entrevistas con el personal asistencial y administrativo que toma decisiones y acciones estratégicas, tácticas y operativas.

Con el objetivo de caracterizar el PAD, se llevó a cabo un diagnóstico integral de la gestión logística, el cual incluyó el análisis del portafolio de servicios y de la estructura de la red de servicios. El análisis del portafolio de servicios de pacientes agudos permitió identificar que el PAD ofrece paquetes de servicio con tres niveles de complejidad, la cual está determinada por el número de curaciones y de aplicación de medicamentos que se prescriban. De igual forma, se pudo identificar el grupo etario y el género de la población de pacientes agudos que se atienden en el PAD (véase figura 2). Con base en los registros de pacientes agudos del PAD, se encontró un crecimiento anual sostenido del número de pacientes desde el 2012 hasta el 2016, con un valor promedio de $44,71 \%$ durante los cinco años, lo que evidencia el crecimiento del programa y el número de pacientes atendidos con servicios de HHC.

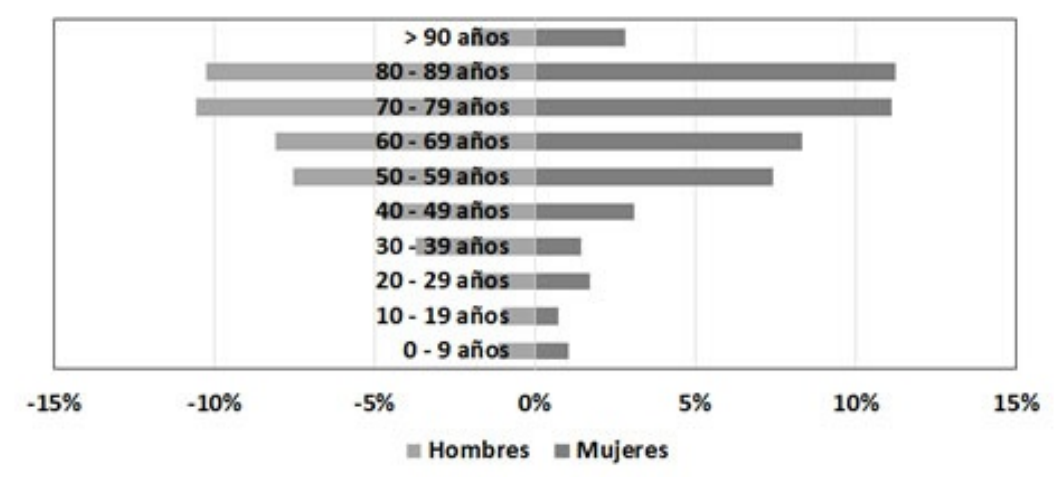

FIGURA 2

Pirámide poblacional de los pacientes atendidos en el servicio de HHC del caso de estudio Fuente: elaboración propia. 
En el análisis de la estructura de la red de servicios, se hizo un análisis geoestadístico de los pacientes y de los servicios de HHC, mediante una georreferenciación de los pacientes en el valle de Aburrá, y una estimación de la carga de trabajo hecha en función de la complejidad de los paquetes de servicio, los tipos de pacientes y los tipos de procedimientos médicos que ofrece el PAD. La figura 3 ilustra la georreferenciación de los pacientes, en la parte (a), y la distribución de la carga de trabajo según el análisis estadístico de los registros de visitas del año 2015, en la parte (b). Como evidencia el análisis, la mayor proporción de la población de pacientes del PAD y de las cargas de trabajo de visitas se encuentra localizada en las zonas nororiental y noroccidental del valle de Aburrá, en concordancia con el régimen de la población atendida en el programa y la distribución socioeconómica de la población en dicha región.

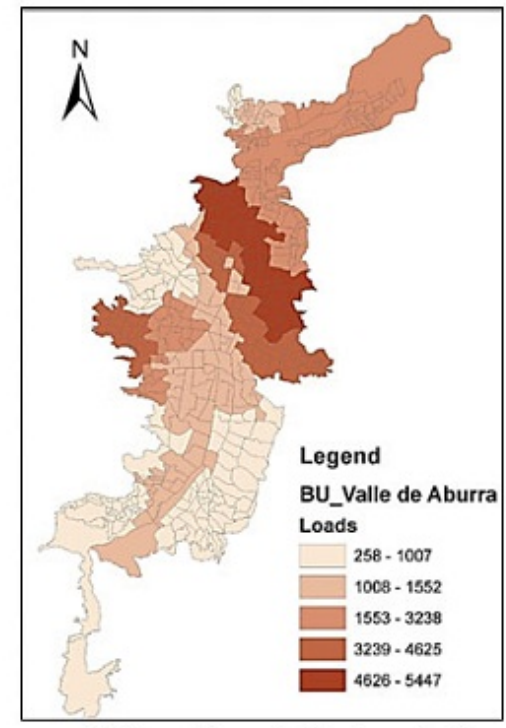

(a) Georreferenciación de pacientes

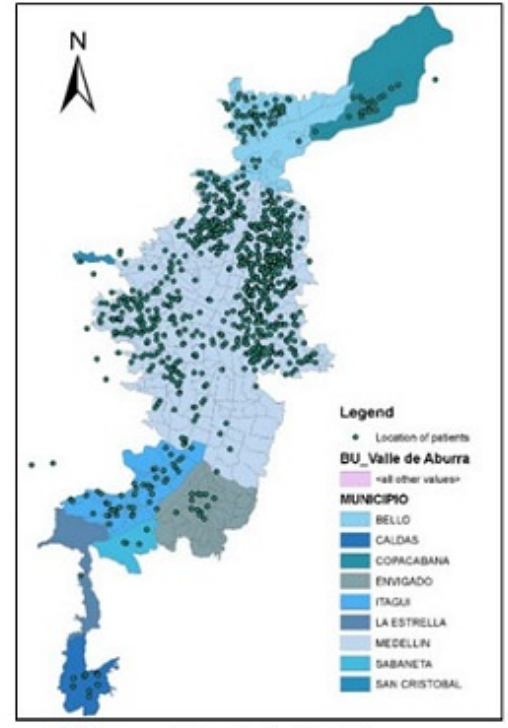

(b) Cargas de trabajo de visitas

FIGURA 3

Análisis geoestadístico de pacientes y de cargas de trabajo en el servicio de HHC del caso de estud Fuente: elaboración propia.

\section{Resultados}

La implementación del modelo de madurez de capacidades logísticas para servicios de HHC se realizó en un caso de estudio con el Programa de Atención Domiciliaria (PAD) que presta la IPS de la Universidad de Antioquia (UdeA) en Colombia. La implementación se hizo en tres etapas. Primero, se realizó una caracterización del caso de estudio mediante un diagnóstico integral de la gestión logística, el cual incluyó el análisis del portafolio de servicios, y de la estructura de la red de servicios. Segundo, se implementó el modelo de madurez mediante la aplicación del cuestionario estructurado y la evaluación de las variables de los procesos logísticos y de servicio. Tercero, se hizo el respectivo análisis de validación de los resultados del modelo, en conjunto con el personal asistencial y administrativo del caso de estudio.

El PAD de la IPS del caso de estudio presenta una madurez global promedio de 2,97/5,00, lo que evidencia las oportunidades de mejoramiento del servicio (véase figura 4). De la madurez global se resalta el hecho de que los procesos del servicio presentan una madurez promedio de 4,02/5,00, mientras que los procesos logísticos presentan una madurez promedio de 1,40/5,00. Adicionalmente, en el interior de los procesos logísticos, se observa que procesos como el diseño de la red y la gestión del transporte requieren más atención por parte de la gerencia del PAD. En la figura 5 se ilustra el mapa de madurez obtenido mediante la interfaz 
gráfica, en el que se reitera cómo los procesos de servicio tienen mayores niveles de madurez, mientras que los procesos logísticos presentan niveles de madurez bajos y, por ende, oportunidades de mejora.

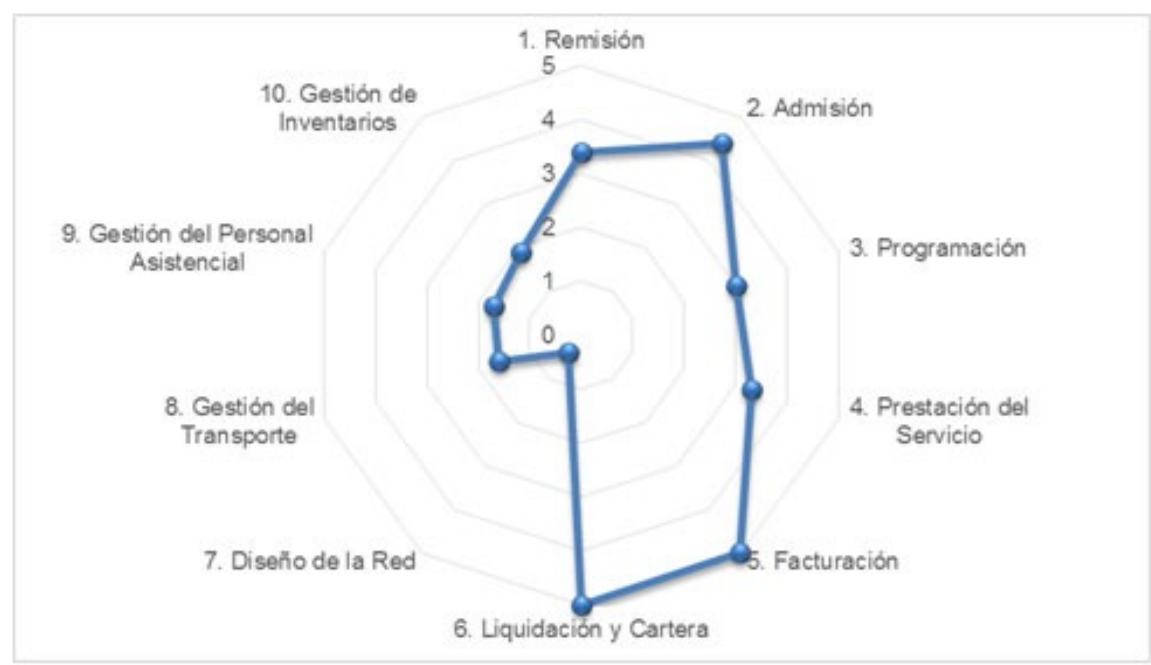

FIGURA 4

Niveles de madurez de las capacidades logísticas del caso de estudio Fuente: elaboración propia.
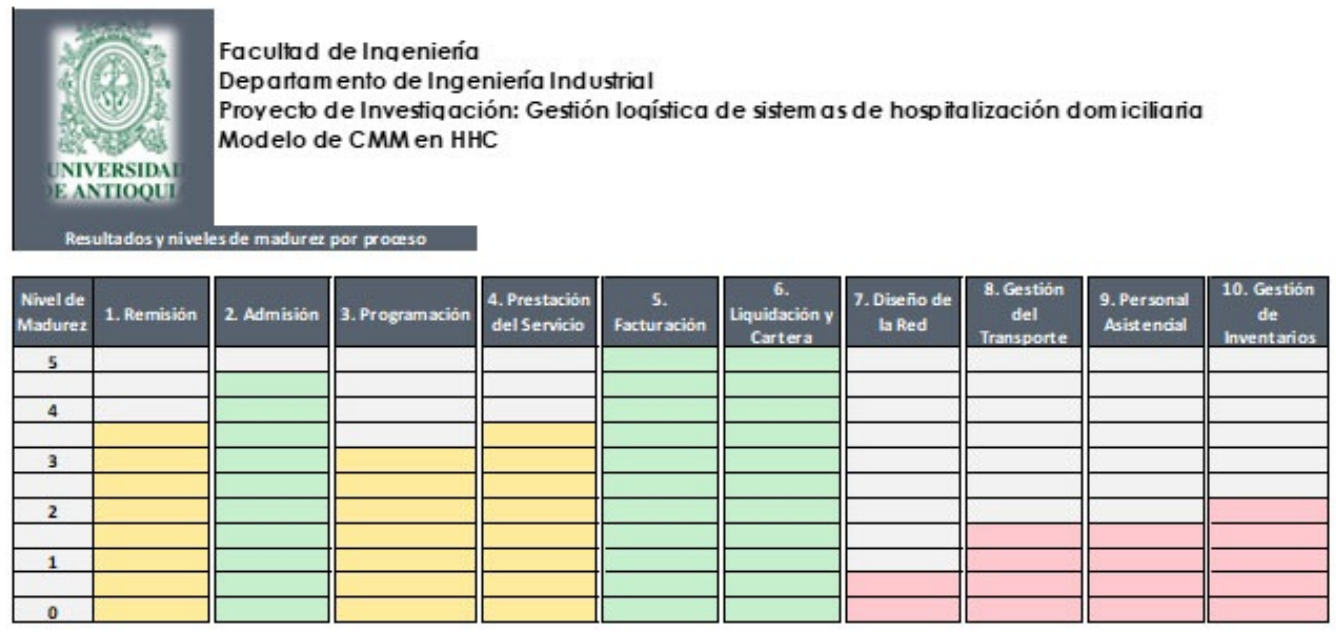

FIGURA 5

Mapa de madurez de las capacidades logísticas del caso de estudio Fuente: elaboración propia.

\section{Resultados de capacidades logísticas}

\section{Procesos logisticos del caso de estudio}

Con una madurez promedio de 1,40/5,00, los procesos logísticos representan la mayor oportunidad de mejora en el caso de estudio. Como se observa en la figura 5, el proceso con el nivel de madurez más bajo es el diseño de la red. Este resultado es acorde con los hallazgos de investigaciones previas en contextos similares (6), así como con la evidencia que muestra que las decisiones estratégicas son las menos estudiadas en el desarrollo de métodos cuantitativos en servicios de salud $(9,17,33)$. Típicamente, las decisiones de localización de centrales de operaciones de HHC y de zonificación de las áreas de cobertura obedecen a 
procesos empíricos, y pocas veces se utilizan métodos cuantitativos que permitan incorporar de manera sistemática, las condiciones operativas propias de la prestación del servicio.

De igual forma, los procesos logísticos de gestión del transporte, del personal asistencial y de los inventarios evidencian oportunidades de mejora, con niveles de madurez de 1,6, 1,7, y 1,9, respectivamente. Parte de estos resultados se explica por la tercerización parcial que hace el PAD en el proceso de prestación del servicio y por la gestión centralizada que hace la IPS de los inventarios de medicamentos, insumos y equipos. Sin embargo, una mejor gestión de la flota vehicular y de la programación y asignación de visitas, así como una mejor estimación de la capacidad del PAD en términos del personal asistencial, prevalecen como puntos clave para la mejora de la madurez en la prestación del servicio.

Para apoyar las decisiones en dichos procesos, existen metodologías que mediante la combinación de métodos cualitativos y cuantitativos pueden generar mejoras en sus niveles de madurez. Para mejorar los procesos de diseño de la red, se han desarrollado trabajos que apoyan las decisiones de zonificación de las áreas de cobertura del servicio. En dichos trabajos se propone la formulación de modelos matemáticos que, resueltos de manera exacta o aproximada, involucran criterios que permiten reducir las distancias recorridas por el personal y balancear las cargas de trabajo $(31,34,35,36,37)$. La gestión del transporte, y en particular las decisiones operativas del diseño de rutas, es el área más estudiada en la literatura de métodos cuantitativos (9). Existen alrededor de veinte trabajos publicados que ilustran cómo mediante el desarrollo de modelos matemáticos y de herramientas de apoyo a la decisión, se puede mejorar la asignación y secuenciación del personal asistencial, con base en los modelos de diseño de rutas.

Para la gestión del personal asistencial, y en particular para la determinación de la capacidad del sistema, se puede también hacer uso de modelos que involucran los tipos de contratación del personal, los tiempos dedicados a viajar entre los domicilios de los pacientes, y aspectos laborales como los turnos de trabajo, las agendas médicas o las curvas de aprendizaje del personal (38). En cuanto a la gestión de inventarios en servicios de HHC, en la literatura se ha reportado un trabajo que ilustra el desarrollo de un modelo para la producción y distribución de medicamentos de tratamientos oncológicos (18). Como es evidente, existen oportunidades de mejora para la madurez de los procesos logísticos que pueden ser abordadas mediante el estudio, el diseño y la implementación de métodos que, basados en los desarrollos existentes, incluyan las condiciones particulares de los sistemas de HHC.

\section{Procesos de servicio del caso de estudio}

Con una madurez promedio de 4,02/5,00, los procesos del servicio evidencian la fortaleza que tiene el PAD en esta gestión del servicio. La implementación del modelo permitió evidenciar que el PAD cuida de manera detallada los procesos de remisión y admisión del paciente, en tanto que es la misma IPS la que remite los pacientes, y estos no se ingresan al programa hasta que no haya una verificación de los criterios de inclusión médicos y locativos, los cuales incluyen también la disponibilidad de la familia para el cuidado del paciente en su domicilio. El proceso de programación de visitas es el que tiene el nivel de madurez más bajo del grupo $(3,00 / 5,00)$, lo cual se comprobó en parte con los resultados del proceso logístico de gestión del transporte. Como se indica en la escala de madurez, el nivel 3,00 señala que el proceso es definido y se encuentra estandarizado y documentado. Sin embargo, hoy la ejecución del proceso se realiza de forma manual por personal asistencial, y no se tiene una herramienta que involucre de manera integral los criterios médicos o geográficos, las características de los pacientes o del personal asistencial. La combinación de dichos criterios resulta en una decisión de tipo operativa compleja, para la cual en la literatura existe un conjunto amplio de metodologías que permiten mejorar su desempeño (32).

Como se observa en la figura 5 , los procesos de facturación, liquidación y cartera son los que tienen el grado de madurez máximo $(5,0 / 5,0)$. Como se muestra en la escala de madurez, la IPS caso de estudio gestiona estos procesos haciendo uso herramientas de tecnología de la información para hacer un control de las métricas de 
desempeño del proceso. Parte de estos resultados se explica por el desarrollo propio de un ERP (enterprise resource planning), como software para la gestión de los procesos de la institución, en el cual se ha incluido módulos para dichos procesos de servicio.

De la misma forma en que la IPS caso de estudio ha realizado esfuerzos para lograr altos niveles de madurez en procesos de servicio, especialmente en los que tienen que ver con procesos administrativos como la facturación, la liquidación y la cartera, los resultados muestran que existe una oportunidad de mejora en otros procesos de servicio, la cual puede ser abordada mediante esfuerzos similares. De manera similar a lo encontrado en los procesos logísticos relativos a la gestión del transporte y del personal asistencial, y en particular las decisiones operativas del diseño de rutas, existen trabajos publicados que ilustran cómo mediante el desarrollo de modelos matemáticos y de herramientas de apoyo a la decisión, es posible mejorar dichos procesos (32). Como se reporta en los trabajos en la literatura, el desarrollo de estas herramientas implica un esfuerzo en la gestión de los procesos, en especial en lo que tiene que ver en los registros del perfil epidemiológico de la población atendida (tipos de pacientes, edad, género, prescripción médica, tipos, frecuencia y cantidad de visitas, entre otros), definición clara del conjunto de actividades por realizar en la ejecución de los procedimientos médicos y registro de los aspectos geográficos del sistema de HHC, en lo que tiene que ver con direcciones de domicilios e información de las mallas viales de acceso.

Los resultados de este caso de estudio pueden contrastarse con trabajos similares. Sin embargo, y como se evidencia en la literatura, si bien existen publicaciones que definen las necesidades y los problemas logísticos de los servicios de HHC $(9,15,16)$, aún son pocos los trabajos que permiten definir y evaluar el grado de madurez en que se encuentran las capacidades logísticas en la atención domiciliaria de los pacientes. En la literatura, se reporta que el primer estudio de la gestión logística de IPS que prestan servicios de $\mathrm{HHC}$ en Colombia es el que se presenta en (6). En dicho estudio, aplicado a 12 IPS que representaban en el 2014 el 39\% de las instituciones habilitadas en el Valle del Cauca para prestar servicios de HHC, se encontró que la madurez promedio de seis procesos de servicio era de 3,2/5,0, y que la IPS con mayor madurez tenía una evaluación de 4,0/5,0. Estos resultados son consistentes con los encontrados en el caso de estudio de este trabajo, en particular por el desarrollo propio del ERP por el PAD de la IPSUdeA. De igual forma, los resultados evidencian que el PAD evaluado presenta un nivel de madurez competitivo en sus procesos de servicio. Por otro lado, el estudio presentado en (6) evidenció que los niveles de madurez promedio para los cuatro procesos logísticos evaluados es de 2,1/5,0. Tal resultado permite, por una parte, confirmar que el nivel de madurez de los procesos logísticos evaluados en el caso de estudio de este trabajo es consistente con los niveles encontrados en estudios previos. Los resultados también permiten confirmar la hipótesis planteada en este trabajo: la evidencia muestra que las IPS habilitadas para prestar servicios de HHC en Colombia toman las decisiones logísticas asociadas a la prestación del servicio de manera empírica y pocas veces se utilizan métodos cuantitativos para apoyarlas de manera sistematizada. Esto se pudo comprobar al encontrar altos niveles de madurez en sus procesos de servicios, así como bajos niveles de madurez en los procesos logísticos. Este hallazgo constituye una oportunidad de trabajo. Como evidencia la literatura, es posible aplicar métodos cuantitativos estructurados para apoyar las decisiones logísticas y mejorar la prestación del servicio.

\section{Conclusiones}

En este trabajo se ha presentado el diseño y la implementación de un modelo de madurez de capacidades logísticas en servicios de HHC. El diseño del modelo se basa en el Capability Maturity Model (CMM), e incluye la evaluación del nivel de madurez de cuatro procesos logísticos y seis procesos de servicio. La implementación del modelo se realizó en un caso de estudio con el Programa de Atención Domiciliaria (PAD) que presta la IPS de la Universidad de Antioquia (UdeA) en Colombia. 
El diseño y la implementación del modelo permitieron identificar brechas tecnológicas y posibles caminos de mejoramiento. De igual forma, el desarrollo de este trabajo permite esbozar tres conclusiones principales. Primero, la revisión de la literatura para el desarrollo del modelo permitió evidenciar que existe un conjunto de autores y trabajos que han estudiado el diseño y el uso del modelo de madurez de capacidades en diferentes contextos desde la década de 1990, y su aplicación más frecuente se encuentra en la industria del diseño de software. De igual forma, existe un conjunto de autores y trabajos que han estudiado el diseño de métodos cuantitativos para el apoyo a decisiones de tipo logístico en servicios de HHC desde la década del 2000, y el problema logístico más estudiado es el diseño de rutas para la asignación y secuenciación de visitas al personal asistencial. Sin embargo, a pesar de los trabajos reportados en la literatura, se encuentran también evidencias previas que muestran cómo la madurez de las capacidades logísticas en servicios de atención médica domiciliaria en el contexto colombiano ha sido estudiada en pocos trabajos, $\mathrm{y}$ existe una brecha tecnológica entre el estado del arte y el nivel de desarrollo en dichas capacidades de las instituciones habilitadas para prestar los servicios de HHC en el país.

Segundo, la implementación del modelo en un caso de estudio real permitió caracterizar el nivel de madurez de las capacidades logísticas de una institución habilitada para la prestación de servicios de HHC en Antioquia, Colombia. El modelo fue implementado en la línea de pacientes agudos, y la mayoría de ellos pertenece al régimen subsidiado de salud. Los resultados de la implementación evidenciaron fortalezas en los procesos de servicio, especialmente en el proceso de admisión de pacientes, y en los relativos a los procesos administrativos de facturación, liquidación y cartera. Estos procesos administrativos son gestionados desde un desarrollo propio de un ERP de la IPS, y por tanto presentan niveles de madurez de 4,02/5,00. Los resultados mostraron también las oportunidades de mejoramiento en los procesos logísticos, especialmente en los relativos al diseño de la red y a la gestión del transporte. La evidencia mostró que las decisiones de dichos procesos se toman de manera empírica y no se hace uso de métodos cuantitativos para apoyarlas de manera sistematizada.

Tercero, lo reportado en la literatura y los hallazgos de este trabajo permiten identificar posibles caminos de mejoramiento en las capacidades logísticas en servicios de HHC. Es posible lograr mejoramientos en los procesos del diseño de la red, mediante la implementación de modelos que apoyen las decisiones de zonificación de las áreas de cobertura del servicio. Apoyar la decisión de dividir el área de cobertura en distritos o zonas con métodos cuantitativos permite asignar efectivamente los recursos disponibles a los distritos que se diseñen, balanceando las cargas de trabajo del personal asistencial y disminuyendo las distancias recorridas para visitar los pacientes.

Aplicar el modelo de madurez de capacidades a una institución que preste los servicios de atención médica domiciliaria permite identificar, en primer lugar, cuáles procesos tienen niveles de madurez altos o bajos. En segundo lugar, a partir de los resultados del modelo, la institución puede priorizar en cuáles procesos requiere mejorar su nivel de madurez desde la gestión del proceso de acuerdo con sus necesidades, o partiendo de los procesos con niveles de madurez más bajos. El objetivo es ir superando los niveles de la escala de madurez gradualmente, pasando del nivel inexistente al inicial, del inicial al repetible, luego al definido y así sucesivamente, hasta que todos los procesos alcancen el nivel de madurez optimizado. Esto permite mejorar continuamente y de forma sistemática los procesos, eliminado las fallas, usando herramientas tecnológicas e innovando para ser competitivo en el mercado.

Entre las oportunidades de investigación, se identifica el interés por definir caminos de mejoramiento para implementar metodologías cualitativas y cuantitativas que permitan incrementar el nivel de madurez de las capacidades logísticas de las instituciones. En ocasiones, esta es la parte más compleja, ya que la implementación de metodologías de mejoramiento como las identificadas en este trabajo requiere constancia, disciplina y compromiso, no solo de las personas a cargo del servicio, sino también de las personas que ejecutan y realizan las operaciones diarias del sistema. Implementar metodologías cualitativas y cuantitativas que de manera sistemática brinden apoyo a la toma de decisiones logísticas, puede mejorar la productividad 
general del sistema, el monitoreo del desempeño enfocado a la generación de valor agregado y a los resultados incluyendo metas alcanzables. Finalmente, todas estas acciones deben estar encaminadas a constituir sistemas de prestación de servicios más robustos que permitan mejorar la atención a los pacientes, y por ende garantizar su acceso al derecho básico de la salud.

\section{Agradecimientos}

Este artículo se deriva del proyecto de investigación Gestión logistica en sistemas de hospitalización domiciliaria, con código PRV16-1-03, desarrollado por el grupo de investigación Ingeniería y Sociedad de la Universidad de Antioquia. Medellín, Colombia.

\section{Referencias bibliográficas}

1. Cotta R, Morales M, Llopis A, Cotta J, Ramón E, Días J. La hospitalización domiciliaria: antecedentes, situación actual y perspectivas. Am J Public Health. 2001;10(1):45-55.

2. Minardi R, Cotta M, Morales Suárez-Varela M, Sette J, Filho C, González AL, et al. La hospitalización domiciliaria ante los cambios demográficos y nuevos retos de salud. Rev Panam Salud Publica/Pan Am J Public Heal. 2002;11(4).

3. Piñarte-Suárez DI, Reinoso-Martínez NV. Atención domiciliaria en salud realizada por instituciones prestadoras de salud en la ciudad de Bogotá y su relación con la atención primaria en salud. Bogotá: Pontificia Universidad Javeriana; 2013.

4. Sierra-Bernal D, Díaz-Arias MA. Plan de negocios atención domiciliaria en salud. Bogotá: Universidad de la Sabana; 2010.

5. Núñez J, Zapata JG, Castañeda C, Fonseca M. La sostenibilidad financiera del sistema de salud colombiano Dinámica del gasto y principales retos de cara al futuro. Bogotá: Fedesarrollo; 2012.

6. Gutiérrez EV, Galvis OD, López DA, Mock-Kow JS, Zapata I, Vidal CJ. Hospital-home health care logistics management in Valle del Cauca: Characterization and diagnosis. Estud Gerenciales. 2014;30(133):441-50.

7. Restrepo C, Echavarría JC, Manrique R, Valencia JE. Costo de hospitalización domiciliaria como alternativa de la hospitalización institucional. Rev CES Med. 2009;23(1).

8. Colombia, Ministerio de Protección Social. Registro Especial de Prestadores de Servicios de Salud [Internet]. 2017. Disponible en: https://www.prestadores.minsalud.gov.co/habilitacion/

9. Gutiérrez EV, Vidal CJ. Home health care logistics management problems: A critical review of models and methods. Rev Fac Ing Univ Antioquia. 2013;68:160-75.

10. Klaassen B, van Beijnum BJF, Hermens HJ. Usability in telemedicine systems-a literature survey. International Journal of Medical Informatics. 2016;93:57-69.

11. Robinson C, Gund A, Sjöqvist BA, Bry K. Using telemedicine in the care of newborn infants after discharge from a neonatal intensive care unit reduced the need of hospital visits. 2016;105(8):902-9.

12. Smith-Strøm H, Iversen MM, Graue M, Skeie S, Kirkevold M. An integrated wound-care pathway, supported by telemedicine, and competent wound management - Essential in follow-up care of adults with diabetic foot ulcers. Int J Med Inform. 2016;94:59-66.

13. Colombia, Ministerio de Protección Social. Resolución 2003 de 2014, por la cual se definen los procedimientos y condiciones de inscripción de los prestadores de servicios de salud y de habilitación de servicios de salud.

14. Bashir B, Chabrol M. Literature review in home care. En: 9th International Conference of Modeling, Optimization and Simulation - MOSIM'12. Bordeaux, France, 2012.

15. Gutiérrez EV. Home health care logistics management problems: an integrated approach to support decisions with hierarchical interdependencies. Cali: Universidad del Valle; 2014. 
16. Gutiérrez EV, Vidal CJ. Home health care logistics management: framework and research perspectives. Int J Ind Eng Manag. 2013;4(3):173-82.

17. Turner J, Mehrotra S, Daskin MS. Perspectives on health-care resource management problems. En: Sodhi MS, Tang CS, editores. A long view of research and practice in operations research and management science: the past and the future [Internet]. Boston, MA: Springer US; 2010 [citado 2011 ago. 18]. p. 231-47. Disponible en: https:/ /www.springerlink.com/index/10.1007/978-1-4419-6810-4

18. Chahed S, Marcon E, Sahin E, Feillet D, Dallery Y. Exploring new operational research opportunities within the Home Care context: the chemotherapy at home. Health Care Manag Sci [Internet]. 2009 [citado 2011 ago. 22];12(2):179-91. Disponible en: https://www.springerlink.com/content/e63206t167075whh/

19. Perrier N, Langevin A, Campbell JF. A survey of models and algorithms for winter road maintenance. Part III: Vehicle routing and depot location for spreading. Comput Oper Res [Internet]. 2007 [citado 2012 nov. 8];34(1):211-57. Disponible en: https://linkinghub.elsevier.com/retrieve/pii/S0305054805001553

20. Perrier N, Langevin A, Campbell JF. A survey of models and algorithms for winter road maintenance. Part I: system design for spreading and plowing. Comput Oper Res [Internet]. 2006 [citado 2012 nov. 8];33(1):209-38. Disponible en: https://linkinghub.elsevier.com/retrieve/pii/S0305054804001625

21. Ernst AT, Jiang H, Krishnamoorthy M, Sier D. Staff scheduling and rostering: A review of applications, methods and models. Eur J Oper Res. 2004;153:3-27.

22. Cayirli T, Veral E. Outpatient scheduling in health care: a review of literature. Prod Oper Manag. 2003;(4):519-49.

23. Vidal CJ, Goetschalckx M. Strategic production-distribution models\#: A critical review with emphasis on global supply chain models. Eur J Oper Res. 1997;98:1-18.

24. Paulk M, Curtis B, Chrissis MB, Weber C. Capability maturity model for software, Version 1.1. Pittsburgh, Pensilvania: Software Engineering Institute; 1993.

25. Saiedian H, Kuzara R. SEI Capability maturity model's impact on contractors. Computer (Long Beach Calif). 1995;28(1):16-26.

26. Gutiérrez EV, Cortés S, Jaén JS. Modelo de madurez de capacidades en la gestión logística de hospitalización domiciliaria: estado actual y nuevos retos. Medellín, Colombia; 2017.

27. Paulk MC, Curtis B, Chrissis MB. Capability maturity model, Version 1.1. Pittsburgh, Pennsylvania Softw Eng Institute. 1993;

28. Saiedian H, Kuzara R. SEI capability maturity model's impact on contractors. Computer (Long Beach Calif). 1995;28(1):16-26.

29. Brailsford SC, Harper PR, Patel B, Pitt M. An analysis of the academic literature on simulation and modelling in health care. J Simul. 2009;3(3):130-40.

30. Bennett Milburn A. Operations research applications in home healthcare. En: Hall R, editor. Handbook of healthcare system scheduling. Internatio. Boston, MA: Springer; 2012. p. 281-302.

31. Benzarti E, Sahin E, Dallery Y. Operations management applied to home care services\#: Analysis of the districting problem. Decis Support Syst [Internet]. 2013;55(2):587-98. Disponible en: https://dx.doi.org/10.1016/j.dss.2 012.10 .015

32. Fikar C, Hirsch P. Home health care routing and scheduling: a review. Comput Oper Res [Internet]. 2017;77:86-95. Disponible en: https://dx.doi.org/10.1016/j.cor.2016.07.019

33. Brailsford S, Vissers J. OR in healthcare: A European perspective. Eur J Oper Res [Internet]. 2011 [citado 2011 ago. 20];212(2):223-34. Disponible en: https://linkinghub.elsevier.com/retrieve/pii/S0377221710006673

34. Blais M, Lapierre SD, Laporte G. Solving a home-care districting problem in an urban setting. J Oper Res Soc [Internet]. 2003 [citado 2011 nov. 11];54(11):1141-7. Disponible en: https://www.palgrave-journals.com/doi finder/10.1057/palgrave.jors.2601625

35. Bennett AR. Home health care logistics planning. Georgia Institute of Technology; 2010.

36. Gutiérrez EV, Vidal CJ. A home health care districting problem in a rapid-growing city. Rev Ing y Univ. 2015;19(1):87-113. 
37. Cortés S, Gutiérrez EV. Congreso Latino-Iberoamericano de Investigación Operativa Program \& Abstracts. En: Vera J, Garavano P, editores. XVIII Latin-Iberoamerican Conference on Operations Research. Santiago de Chile: Association of Latin-Iberoamerican Operational Research Societies, ALIO; 2016. p. 51-2.

38. Gutiérrez EV. Home health care logistics management problems: an integrated approach to support decisions with hierarchical interdependencies. Cali: Universidad del Valle; 2014.

Licencia Creative Commons CC BY 4.0

Para citar este artículo: Gutiérrez EV, Cortés Zapata S, Jaén Posada JS. Diseño e implementación de un modelo de madurez de capacidades logísticas en servicios de atención médica domiciliaria. Rev. Gerenc. Polit. Salud. 2018;17(35). https://doi.org/10.11144/Javeriana.rgps 17-35.dimm 